\title{
Preliminary proper motion analysis of the Carina dwarf spheroidal
}

\author{
J. L. Carlin ${ }^{1}$, S. R. Majewski, D.I. Casetti-Dinescu, and T. M. Girard \\ ${ }^{1}$ Dept. of Astronomy, Univ. of Virginia, PO Box 400325, Charlottesville, VA 22904-4325 USA
}

\begin{abstract}
We present preliminary results from a proper motion study of the Carina dwarf

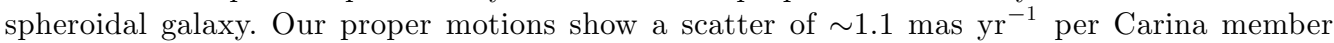
star, and we determinate the mean ensemble motion to an accuracy of $\sim 7$ mas century $^{-1}$. While this is a precise measurement of the relative proper motions of Carina members, our correction to an absolute frame is limited by the small number of measured QSOs in the field.
\end{abstract}

Keywords. galaxies: dwarf, galaxies: individual (Carina dSph), astrometry

\section{Introduction}

The discovery by Muñoz et al. 2006 (M06) of a population of extratidal stars associated with the Carina dwarf spheroidal (dSph) bolsters the case for the importance of tidal disruption in dSph evolution and the formation of the Milky Way halo. Muñoz et al. 2007 explained many observed properties of Carina, including the large inferred M/L ratio, by modeling it as a tidally disrupting $\mathrm{dSph}$. However, the absolute proper motion (PM) is necessary to derive an orbit and assess the importance of tidal forces for Carina. The only existing measurement of the space motion of Carina (Piatek et al. 2003) utilized only two HST fields centered on known QSOs. We will measure the absolute PM for Carina using photographic plates covering a much larger area than the HST observations.

\section{Data and measurements}

Our dataset consists of 41 CTIO Blanco 4m Prime Focus Camera photographic plates (plate scale $18.60^{\prime \prime} / \mathrm{mm}$ ), spanning the period 1984 - 1998. The plates were digitized in April 2007 using the USNO StarScan system, which produces typical measurement errors of $\sim 0.3 \mu \mathrm{m}$, with better than $0.2 \mu \mathrm{m}$ repeatability (Zacharias et al. 2004). Proper motions were measured using similar techniques as in, e.g., Girard et al. 1989, using polynomial geometric terms, and linear color and magnitude terms in the plate solutions.

Carina stellar populations are easily discernible in the CMD (from the photometric catalog of M06) shown in Figure 1(a), from which we have selected a sample of candidate Carina member stars (grey diamonds). Confirmed Carina radial velocity (RV) members (M06) are shown as large filled black circles, and QSOs from Véron-Cetty \& Véron (2006) as asterisks. The QSOs will be used as our tie to an absolute, "fixed" reference frame, and include the two QSOs used in the Piatek et al. 2003 HST study.

\section{Measured proper motions}

Proper motions along the two spatial dimensions $(\mathrm{x}, \mathrm{y} \sim \alpha, \delta)$ show (Figure $1(\mathrm{~b})$ ) that Carina stars clearly separate from the field (mainly foreground thin/thick disk dwarfs), exhibiting extremely small motion (similar to the "fixed" QSOs) expected for a dwarf galaxy at $\sim 100 \mathrm{kpc}$ (Mateo (1998)). Table 1 shows sigma-clipped mean PMs for each 

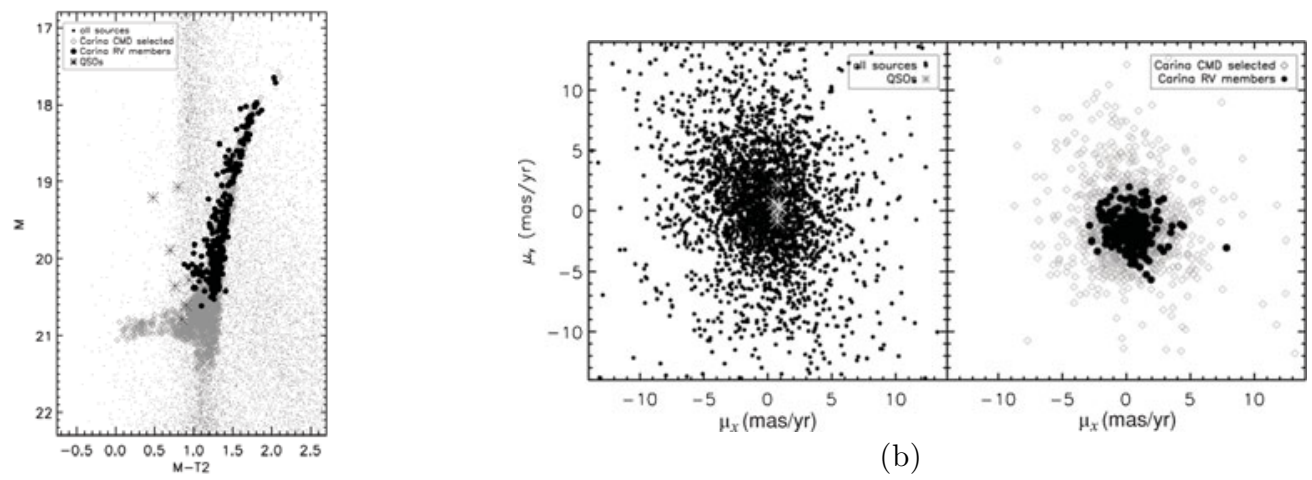

(a)

Figure 1. (a) Washington M, T2 ( V , I) CMD of the Muñoz et al. 2006 sample, with known QSOs as large asterisks. Large filled circles represent confirmed Carina RV members (Muñoz et al. 2006), and grey diamonds show our CMD selection. (b) Relative proper motions (in mas/yr). Left: all measured stars, with QSOs as grey asterisks. Right: CMD-selected Carina candidates (grey diamonds), with confirmed Carina RV members (black circles). Note the tight clumping of the RV sample, indicative of a well-measured mean motion for Carina.

Table 1. Relative proper motion measurements (all units mas/yr).

\begin{tabular}{l|c|c|c|c|c|c|c|}
\hline Sample & $\#$ & $\bar{\mu}_{x}$ & $\bar{\mu}_{y}$ & $\sigma_{\mu_{x}}$ & $\sigma_{\mu_{y}}$ & $\sigma_{\bar{\mu}_{x}}$ & $\sigma_{\bar{\mu}_{y}}$ \\
\hline RV members & 269 & 0.344 & -1.634 & 1.003 & 1.211 & 0.061 & 0.073 \\
CMD-selected & 1416 & 0.379 & -1.424 & 1.640 & 1.862 & 0.043 & 0.049 \\
QSOs & 7 & 0.351 & -0.724 & 0.941 & 0.918 & 0.356 & 0.347 \\
\hline
\end{tabular}

of the three samples. The purest Carina sample (the RV members), has per-star errors of $\sigma_{\mu_{x, y}} \sim 1.1 \mathrm{mas} / \mathrm{yr}$. From the RV members, we calculate a mean bulk motion of the Carina dSph accurate to $\sim 6.5$ mas/century. The CMD-selected sample shows larger standard deviations in individual measurements, as many of these stars are near the faint limit of the survey. Clearly the accuracy of our absolute PM for Carina will be dominated by the correction to an absolute frame (using QSOs and galaxies), not the measurement precision of the Carina PM center. Further work at identifying well-measured (point-like) galaxies in the field will improve our correction to absolute proper motions. In addition, we are working to address remaining magnitude and color trends in the preliminary PMs, which should reduce the scatter in the reference QSO measurements.

\section{References}

Girard, T. M., Grundy, W. M., Lopez, C. E., \& van Altena, W. F. 1989, AJ, 98, 227

Mateo, M. L. 1998, ARAA, 36, 435

Muñoz, R. R., et al. 2006, ApJ, 649, 201 (M06)

Muñoz, R. R., Majewski, S. R., \& Johnston, K. V. 2007, ApJ, submitted

Piatek, S., Pryor, C., Olszewski, E. W., et al. 2003AJ, 126, 2346

Véron-Cetty, M.-P., \& Véron, P. 2006, A\& A, 455, 773

Zacharias, N., Urban, S. E., Zacharias, M. I., et al. 2004, AJ, 127, 3043 